\title{
Gallbladder Necrosis, CTCAE 5.0
}

National Cancer Institute

\section{Source}

National Cancer Institute. Gallbladder Necrosis, CT CAE 5.0. NCI Thesaurus. Code C146735.

A disorder characterized by a necrotic process occurring in the gallbladder. 